\title{
Rezension: Thierry Urwyler, Das Teilnahmerecht der Verteidigung am Explorationsgespräch des psychiatrischen Sachverständigen mit der beschuldigten Person im Lichte der EMRK
}

Stephan Bernard *

Thierry Urwyler diskutiert in seiner Luzerner Dissertation die strittige strafprozessuale Frage, ob die Verteidigung am Explorationsgespräch des psychiatrischen Sachverständigen mit der beschuldigten Person teilnehmen darf, sowie ob und gegebenenfalls wie Explorationsgespräche zu dokumentieren sind. Ein besonderes Augenmerk richtet er dabei auf die Vorgaben der Europäischen Menschenrechtskonvention. Im Gegensatz zum Bundesgericht, welches jedenfalls im Regelfall kein Teilnahmerecht der Verteidigung zulassen will, spricht sich der Autor für ein Teilnahmerecht und für eine audiovisuelle Aufzeichnung des Explorationsgesprächs aus.

I. Ausgangslage: Die Rolle des psychiatrischen Sachverständigen im

Übergang vom Schuld- zum Präventionsstrafrecht. .274

II. Kontrolle der psychiatrischen Expertise durch die Verteidigung?....................274

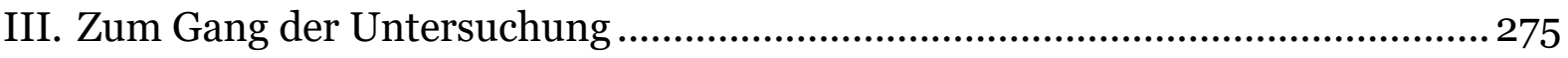

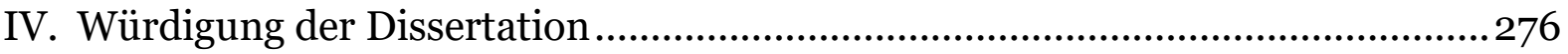

Zitierung: $\quad$ Stephan Bernard, Rezension: Thierry Urwyler, Das Teilnahmerecht der Verteidigung am Explorationsgespräch des psychiatrischen Sachverständigen mit der beschuldigten Person im Lichte der EMRK, in: sui-generis 2020, S. 273

URL: $\quad$ sui-generis.ch/136

DOI: $\quad$ https://doi.org/10.21257/sg.136

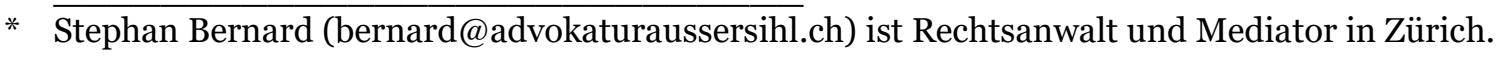

Dieses Werk ist lizenziert unter einer Creative Commons Namensnennung - Weitergabe unter gleichen Bedingungen 4.0 International Lizenz. 


\section{Ausgangslage: Die Rolle des} psychiatrischen Sachverständigen im Übergang vom Schuld- zum Präventionsstrafrecht

Seit über einem Jahrzehnt lässt sich ein Umbau des reaktiven Strafrechts in ein strafendes Präventionsrecht beobachten. ${ }^{1}$ Insbesondere stationäre therapeutische Massnahmen nach Artikel 59 StGB nehmen markant zu. Sie können ad infinitum verlängert werden und dauern oft länger als die schuldangemessene Strafe. Über die Verhängung oder Fortsetzung der Massnahme entscheidet nicht primär die Tat, sondern das Profil der Täter_innen. In den Verfahren zur Anordnung oder Verlängerung solcher Massnahmen kommen Expertisen von psychiatrischen Sachverständigen eine Schlüsselrolle zu. Sie liefern die Grundlage, die vom Gericht in Entscheiden vielfach faktisch übernommen wird. ${ }^{2}$

\section{Kontrolle der psychiatrischen Expertise durch die Verteidigung?}

2 Seit knapp zehn Jahren findet im Schrifttum eine Debatte statt, ob und inwiefern es der Verteidigung möglich ist, die Genese und das Resultat psychiatrischer Expertisen effektiv zu kontrollieren bzw.

\footnotetext{
Statt vieler prägnant Marcel Alexander Niggli, Strafrecht: Von der Repression zur Prävention, plädoyer 2/2014, S. 28 ff.; derselbe, Vom Repressions- zum Präventionsstrafrecht: Die Abkehr von der Ahndung begangener hin zur Verhinderung befürchteter Delikte, in: Forum Strafverteidigung et al. (Hrsg.), Strafverteidigung und Sicherheitswahn, Wien u.a. 2014, S. $13 \mathrm{ff}$.

2 Hierzu der Essay des Rezensenten, Freiheitsentziehendes Massnahmenrecht oder freiheitsentziehende Massnahmen jenseits des Rechts?, in: André Kuhn/Christian Schwarzenegger/Joëlle Vuille (Hrsg.), Schweizerische Arbeitsgruppe für Kriminologie, Band 35, Strafverfolgung - Individuum - Öffentlichkeit im Spannungsfeld der Wahrnehmungen, Bern 2017, S. $139 \mathrm{ff}$.
}

was für eine Kontrolle vonnöten wäre. Urwyler zeichnet diese Diskussion in der Aufarbeitung des Forschungsstandes detailliert nach (S. 27 ff.). 3 Der Kern dieser Diskussion bildet die Frage, wie psychiatrische Explorationsgespräche zu dokumentieren sind und ob der Verteidigung ein Teilnahmerecht am Explorationsgespräch der beschuldigten Person zuzubilligen ist. Während das Bundesgericht ein Teilnahmerecht jedenfalls im Grundsatz ablehnt, mehren sich in der Literatur die Stimmen, die sich dafür aussprechen (zum Ganzen S. 27 ff.). Zumindest hat das Bundesgericht ein Teilnahmerecht nicht vollständig ausgeschlossen und für Ausnahmen vom Regelfall die Türe einen Spaltbreit offengelassen. 4

3 Die Dissertation Urwylers hinterfragt den Standpunkt des Bundesgerichts insbesondere im Lichte der Europäischen Menschenrechtskonvention. Der Autor kommt zum Ergebnis, dass unter den bestehenden verfahrensrechtlichen Bedingungen mangels effektiven Kontrollmöglichkeiten der Verteidigung kein faires Verfahren im Sinne von Art. 6 EMRK gewährleistet werde: «Nimmt man die beschuldigte Person als Verfahrenssubjekt ernst, muss ein faires Verfahren im Rahmen der Schuldfähigkeits- und Mas-

3 Die wissenschaftliche Redlichkeit gebietet es, offenzulegen, dass der Rezensent sich in verschiedenen Veröffentlichungen stets für ein Teilnahmerecht der Verteidigung ausgesprochen hat: Neue StPO: Nach wie vor keine Kontrolle der sogenannten Erhebung bei (psychiatrischen) Begutachtungen?, Anwaltsrevue 1/2011, S. 9 ff. (gemeinsam mit Anja Martina Binder); Sicherheitsgesellschaft und psychiatrische Begutachtungspraxis in Strafverfahren, Jusletter vom 13. Februar 2012 (Sondernummer zur Strafprozessordnung); Psychiatrische Gutachter ohne strafprozessuale Kontrolle?, ZStrR 1/2015, S. 76 ff. (gemeinsam mit Rafael Studer).

4 BGE 144 I 253 E. 3.8. 
snahmenindikationsbegutachtung eine audiovisuelle Aufzeichnung der Exploration und ein Teilnahmerecht der Verteidigung beinhalten» (S. 253, insgesamt die Arbeit zusammenfassend S. 247 ff.).

\section{Zum Gang der Untersuchung}

4 Die Arbeit von Thierry Urwyler fasst den Untersuchungsgegenstand in einer 26seitigen Einleitung konzis zusammen. Sodann wird die gewählte Thematik hinsichtlich der verfassungsrechtlichen und gesetzlichen Ausgangslage erörtert (S. 27 bis 54). Urwyler gibt hier die Argumentation der «teilnahmefeindlichen» und «teilnahmefreundlichen» Standpunkte vor dem Hintergrund der nationalen Gesetzgebung, namentlich Art. 147 StPO, wieder. Im Ergebnis vertritt er die Auffassung, dass die Strafprozessordnung und das eidgenössische Verfassungsrecht keine Basis für ein Teilnahmerecht der Verteidigung bieten und grundsätzlich der teilnahmefeindlichen Auslegung von Art. 147 StPO beizupflichten sei. Während für polizeiliche Einvernahmen ein Teilnahmerecht der Verteidigung nach Art. 147 StPO aus einem expliziten Verweis hervorgehe (Art. 312 Abs. 2 StPO), fehle es für die sachverständige Erhebung gerade daran. Der Wille des Gesetzgebers sei klar, die StPO-Analogie zur Einvernahmenormierung misslinge und eine völker- und verfassungskonforme «Begradigung» in der Auslegung der Strafprozessordnung sei unzulässig (S. $50 \mathrm{ff}$.).

5 In der Folge wendet sich der Autor der Kernthematik seiner Arbeit zu, mithin der Frage, wie das Teilnahmerecht und die audiovisuelle Aufzeichnung im Lichte der Europäischen Menschenrechtskon- vention zu beurteilen sind. Er betritt damit, jedenfalls hinsichtlich der Argumentationstiefe, Neuland. Bisher hat zu dieser Thematik niemand derart eingehend das Augenmerk auf die Rechtsprechung des Europäischen Gerichtshofs für Menschenrechte gerichtet.

6 Präzis gibt Urwyler zunächst das Prüfungsmodell zu Art. 6 EMRK wieder (Verfahrensfairness). Er diskutiert dabei zuerst im «Lichte des Rechts auf Stellungnahme zum Gutachten» sehr sorgfältig die derzeitige Praxis (S. 55 bis 164). Er kommt zum Schluss, dass eine Verletzung von Art. 6 Ziff. 1 i.V.m Art. 6 Ziff. 3 lit. d. vorliege (S. $130 \mathrm{ff}$.). Sodann zeigt der Autor en détail auf, weshalb im Teilnahmerecht der Verteidigung und der audiovisuellen Aufzeichnung die sachgerechte Lösung liege, und warum eine rein auf die Position und Rolle des Sachverständigen ausgerichtete Korrektur nicht ausreiche (zusammenfassend S. 161 ff.).

7 Mit einem analogen Aufbau und Prüfungsschema handelt Urwyler sodann den Gegenstand «im Lichte des Rechts auf Beizug der Verteidigung» ab (S. 165 bis 246). Auch daraus leitet er ab, dass insbesondere das Teilnahmerecht der Verteidigung zwingend sei (S. $240 \mathrm{ff}$. und S. $245 \mathrm{ff}$.). Besonders hervorzuheben ist, dass der Autor sehr präzise darlegt, weshalb teilnahmerechtslose Lösungsansätze mangels ausreichendem Schutzgehalt im Ergebnis ausscheiden (S. 245). Er zeigt dies anhand der möglichen Alternativen in Form des Verbots der Befragung zum Tatsachverhalt (S. 226 ff.), der audiovisuellen Aufzeichnung (S. 228 f.), des Tatinterlokuts (S. 229 ff.), der Fiktion des fehlenden Beweiswertes von Explorationsangaben ausserhalb des Gutachtens 
(S. $231 \mathrm{ff}$.$) und Verwertungsverboten$ (S. 232 ff.).

8

Die Monographie mündet in einer inhaltlich konzisen und sprachlich sehr sorgfältig redigierten Schlussbilanz (S. 247 ff.).

\section{Würdigung der Dissertation}

9 Thierry Urwyler kommt das Verdienst zu, eine zentrale Fragestellung der gegenwärtigen Strafprozessrechtsdogmatik aufgegriffen und akribisch erörtert $\mathrm{zu}$ haben. Dem Autor gelingt darüber hinaus das Kunststück, den anspruchsvollen Gegenstand in einem geradezu perfekten Umfang abzuhandeln. Zwar wurden zentrale Argumente bereits diskutiert, indessen besticht seine Dissertation durch ihre Tiefe und argumentative Sorgfalt. Namentlich der im Zentrum stehende Bezug zur Europäischen Menschenrechtskonvention wurde bislang nirgends so fundiert ausgelotet. Dies verdient schon nur deshalb Anerkennung, weil hierzulande strafprozessuale Abhandlungen unter besonderer Berücksichtigung der Europäischen Menschenrechtskonvention während langer Jahre rar waren und hier weiterhin zahlreiche wichtige Forschungsfelder zu erschliessen sind. Dieses Vorgehen erlaubt Urwyler auch, $\mathrm{zu}$ einer eigenständigen Position zu gelangen: Zwar hält er die «teilnahmefeindliche» Auslegung der Strafprozessordnung an sich für zutreffend und erteilt «teilnahmefreundlichen» Interpretationen eine Absage, spricht sich aber nach dem eingehenden Einbezug der Europäischen Menschenrechtskonvention für eine «teilnahmefreundliche» Praxis direkt abgestützt auf die Konvention aus.
10 Just bei dem Weg zu dieser eigenständigen Auffassung sind indessen die einzigen (methodischen) Vorbehalte $\mathrm{zu}$ den ansonsten durchwegs überzeugenden Ausführungen Urwylers anzubringen: Der Rezensent hegt gewisse Zweifel, ob sich die Indizien im Gesetzgebungsprozess wider ein Teilnahmerecht der Verteidigung effektiv zu einem «klaren Willen des Gesetzgebers» (S. 51 f.) verdichtet haben.

11 Sodann wird nicht näher erörtert, wie sich das Verhältnis der nationalen Rechtsquellen Strafprozessordnung und Verfassung einerseits zur Europäischen Menschenrechtskonvention und andererseits bei der Exegese konkreter Rechtsfragen darstellt. Dies führt zu offenen Fragen hinsichtlich des Auslegungsverständnisses. In der besprochenen Schrift wird in einem ersten Schritt eine völkerrechtskonforme Auslegung von Art. 147 StPO zurückgewiesen, weil sie dem eindeutigen Willen des Gesetzgebers widerspreche. Dies führt zum Ergebnis, dass sich eine teilnahmerechtsfreundliche Auslegung nicht direkt auf die Strafprozessordnung bzw. das schweizerische Verfassungsrecht abstützen lasse (S. 52 f.). Urwyler legt damit die Strafprozessordnung sowie das schweizerische Verfassungsrecht zunächst gleichsam selbstbezüglich aus und denkt die Europäische Menschenrechtskonvention nicht gleichzeitig als parallele Rechtsquelle dazu. Er erörtert die sich aus der Konvention ergebenden Implikationen erst hernach (so explizit zu dieser Abfolge der Untersuchungsetappen S. 25). Erst in diesem zweiten Schritt misst Urwyler den strafprozessualen Status quo an der Europäischen Menschenrechtskonvention und kommt zum Schluss, 
dass direkt gestützt auf diese Rechtsquelle ein Teilnahmerecht der Verteidigung an Explorationsgesprächen und eine audiovisuelle Aufzeichnung geboten sei.

Dagegen kann eingewendet werden, dass sich die völkerrechtskonforme Auslegung einer landesrechtlichen Norm (oder in den Worten Urwylers: «Begradigung») nicht in der angeführten Trennschärfe von der Ableitung eines rechtlichen Ergebnisses direkt gestützt auf das Völkerrecht unterscheiden lässt. Diese Trennung lässt sich nur dann so scharf treffen, wenn beide rechtliche Texturen nicht als gleichzeitig zu denkende Bestandteile einer Rechtsordnung angesehen werden. Völkerrecht kommt bei dieser Argumentation, so letztlich die unausgesprochene Prämisse Urwylers in seinen Untersuchungsetappen (S. 25), zum Landesrecht gleichsam von aussen bzw. als «später zu betrachtend» dazu. Es wird damit mutmasslich als eine Art eigenständiger Rechtskorpus angesehen, der nicht umfassend ins Landesrecht als gleichzeitig geltende Rechtsquelle integriert ist und bei der Interpretation jeder Norm somit von Anfang an gleichrangig mitbedacht werden muss, sondern erst in einem zweiten Schritt und aus sich heraus.

Der zu zahlende juristische Preis dieser Betrachtungsweise wäre im Ergebnis wohl die Aufgabe der Figur der Einheit der Rechtsordnung. Sie führte zu einer gedanklichen Aufteilung in eine Strafprozessordnung, die aus sich heraus zu verstehen ist, und eine Europäische Menschenrechtskonvention, an der sich sodann die strafprozessuale Praxis zu messen hat. Dies mag zwar prima vista wohl in vielen Konstellationen im Endre- sultat zu analogen inhaltlichen Ergebnissen führen wie eine gleichzeitige Auslegung von Landes- und Völkerrecht. Immerhin beschlägt dies aber fundamentale Fragen im Umgang mit parallel geltenden nationalen und völkerrechtlichen Rechtsquellen, deren Bedeutung kaum abnehmen wird und allein deshalb eine nähere Erörterung verdiente. Wollte man diesen Standpunkt zum Verhältnis Landes- und Völkerrecht rechtswissenschaftlich vertreten, müsste dies methodisch daher näher begründet werden.

14 Teilt man diese Auffassung nicht, wofür auf den ersten Blick zumindest die Vorstellung einer einheitlichen Rechtsordnung spricht, ist es denknotwendig unmöglich, Landesrecht ohne umfassende Berücksichtigung des gleichzeitig geltenden und ins Landesrecht inkorporierten Völkerrechts aus sich selbst heraus auszulegen. Bei einem Auslegungsvorgang sind demnach von Anfang an Strafprozessordnung und Europäische Menschenrechtskonvention gleichzeitig in wechselseitigen Bezügen als gemeinsame Bestandteile einer Rechtsordnung als verschiedene und parallel geltende Rechtsquellen zu denken. Dies führt im Resultat dann mutmasslich zu der von Urwyler abgelehnten völkerrechtskonformen «Begradigung», wobei es sich bei Lichte betrachtet deshalb wohl eher um eine systematische Auslegung von Art. 147 StPO unter Berücksichtigung der Europäischen Menschenrechtskonvention handeln dürfte.

15 Insgesamt vermag die Argumentation Urwylers in diesem methodischen Punkt demnach nicht restlos zu überzeugen. Dies ist aber mutmasslich auch und gerade dem Umstand geschuldet, dass eine 
grundlegende Vermessung des komplexen Verhältnisses Strafprozessordnung sowie Verfassungsrecht zur Europäischen Menschenrechtskonvention auf sich warten lässt und sich der Autor damit in rechtlich diffiziles Gelände gewagt hat.

Indessen beschlagen diese Ausführungen aufgrund des letztlich identischen rechtlichen Ergebnisses lediglich Begründungssubtilitäten und bleiben der einzige Kritikpunkt an einer ansonsten makellosen Arbeit. Es ist sehr zu hoffen, dass die Arbeit Urwylers die gebührende höchstrichterliche Beachtung erhält. Das Bundesgericht kommt nach der Auffassung des Rezensenten kaum umhin, sich bei künftigen Entscheiden sehr genau mit Thierry Urwylers Einwänden zu befassen. Möglicherweise kommt es aufgrund der detailliert dargelegten Implikationen der Europäischen Menschenrechtskonvention auf die gefällte Wegmarke hinsichtlich der Teilnahmerechte der Verteidigung am psychiatrischen Explorationsgespräch zurück. Zumindest müsste die offene Frage der Dokumentation durch das Bundesgericht nun wohl definitiv dahingehend geklärt werden, dass sämtliche psychiatrische Explorationsgespräche audiovisuell aufzuzeichnen sind.

Falls das Bundesgericht seinen Standpunkt hinsichtlich des Teilnahmerechts nicht grundsätzlich in Wiedererwägung zieht, darf man nach der Arbeit Urwylers gespannt sein, wann ein Teilnahmerecht im Ausnahmefall im Sinne der bundesgerichtlichen Rechtsprechung zugelassen wird. Folgt man der Argumentation im rezensierten Werk, wäre es dann zu gewähren, wenn die Begutachtung eines Nicht-Geständigen ansteht oder wenn die Begutachtung den Schuldpunkt aus anderen Gründen beeinflussen könnte. In diesen Fällen hat die Exploration auch den Charakter einer Sachverhaltsermittlung, da zwangsläufig Angaben zur Tat erfragt werden. In diesen Fällen müssten die Teilnahmerechte der Verteidigung gelten, nicht zuletzt zur Wahrung des Grundsatzes nemo tenetur. Würden allerdings die Ausnahmen vom Regelfall derart interpretiert, so führte dies im Ergebnis dazu, dass das Verhältnis vom Regel- und Ausnahmefall faktisch in sein Gegenteil verkehrt würde. Thierry Urwylers Standpunkt hätte dann die höchstrichterliche Rechtsprechung zumindest indirekt geprägt.

18 Sollte die Frage nicht auf nationaler Ebene geklärt werden, so ist von grossem rechtswissenschaftlichen wie praktischen Interesse, ob der Europäische Gerichtshof für Menschenrechte der fundierten Auslegung des rezensierten Werks folgt. $\mathrm{Zu}$ wünschen und dem Autor von Herzen zu gönnen wäre es.

«Das Teilnahmerecht der Verteidigung am Explorationsgespräch des psychiatrischen Sachverständigen mit der beschuldigten Person im Lichte der EMRK», Thierry Urwyler, Luzerner Beiträge zur Rechtswissenschaft, 135, Schulthess Verlag, Zürich 2019, ISBN: 978-3-7255-8041-5 (gedruckte Ausgabe, kartoniert, Paperback) 